\title{
Transdermal optical renal function monitoring in humans: development, verification, and validation of a prototype device
}

Martin P. Debreczeny

Richard B. Dorshow 


\title{
Transdermal optical renal function monitoring in humans: development, verification, and validation of a prototype device
}

\author{
Martin P. Debreczeny and Richard B. Dorshow* \\ MediBeacon Inc., St. Louis, Missouri, United States
}

\begin{abstract}
A prototype medical device for monitoring kidney function by transdermal measurement of the clearance rate of the exogenous fluorescent tracer agent MB-102 (administered intravenously) was developed. Verification of the device with an in vitro protocol is described. The expected renal clearance of the agent was mimicked by preparing a dilution series of MB-102 in the presence of a scattering agent. The slope of a linear fit to the logarithm of fluorescence intensity as a function of dilution step agreed with predictions within $5 \%$, a level of accuracy that would be adequate in assessment of GFR to prevent misdiagnosis of kidney disease. Transdermal measurement was validated using a rat model. A two-compartment pharmacokinetic dependence was observed, with equilibration of the fluorescent agent between the vascular space into which it was injected and the extracellular space into which it subsequently diffused. The best observed signal-to-noise ratios were about 150 , allowing determination of the renal clearance time with $5 \%$ precision using a 10-min fitting window. Based on the verification and validation methods for transdermal fluorescence detection described herein, the instrument has been approved by the FDA for a first-in-human clinical study, and a first transdermal clearance curve in a human is presented herein. $\odot$ The Authors. Published by SPIE under a Creative Commons Attribution 3.0 Unported License. Distribution or reproduction of this work in whole or in part requires full attribution of the original publication, including its DOI. [DOI: 10.1117/1.JBO.23.5.057003]
\end{abstract}

Key words: transdermal fluorescence; renal function monitoring; exogenous agent; noninvasive; prototype medical device; MB-102. Paper 170687R received Oct. 21, 2017; accepted for publication Apr. 26, 2018; published online May $11,2018$.

\section{Introduction}

Current clinical practice for renal function monitoring is to calculate estimated glomerular filtration rate (eGFR), using one of the several empirically derived equations with inputs of serum creatinine concentration (requiring a blood draw) and several other parameters including height, weight, gender, and ethnicity. ${ }^{1,2}$ The resulting eGFR suffers from several drawbacks: (1) actual GFR can decrease by as much as $50 \%$ before it is reflected as an abnormal serum creatinine concentration, resulting in a lack of sensitivity, (2) serum creatinine concentration is affected by factors other than renal clearance such as age, muscle mass, and diet, and (3) abnormal serum creatinine concentration may not manifest for up to $72 \mathrm{~h}$ after a renal insult or injury, prohibiting real-time monitoring. ${ }^{3,4}$ It is this latter consequence of eGFR that renders it less than optimal, and often useless, in the intensive care unit (ICU) environment. Dynamic changes in patient's renal function, which often occur in the ICU, cannot be observed with eGFR. Hence, there is an unmet medical need for point-of-care measured (not estimated) GFR.

Monitoring of tracer agents that are cleared exclusively by glomerular filtration have long been used for more accurate GFR determination. However, this has traditionally required numerous blood draws and subsequent laboratory analysis,

*Address all correspondence to: Richard B. Dorshow, E-mail: rbdorshow@ medibeacon.com with the result that such techniques are seldom used in clinical practice, except in a few major centers for kidney treatment. ${ }^{4}$

Efforts to circumvent the need for blood draws and laboratory analysis were first focused on transdermal monitoring of radioactive agents cleared by glomerular filtration. ${ }^{5}$ While technically promising, these techniques were never widely adopted, due to concerns surrounding the handling and routine patientadministration of radioactive materials, especially in the ICU. More recently, efforts have focused on the transdermal monitoring of fluorescent tracer agents that are cleared by glomerular filtration. ${ }^{6-8}$ This would result in an actual measured GFR in real-time at the point-of-care.

In prior animal studies, the fluorescent tracer agent MB-102 has been shown to be nontoxic and cleared exclusively by the kidneys without being metabolized nor secreted or reabsorbed by the renal tubules, making it an ideal vehicle for determining GFR. ${ }^{9,10}$ Transdermal monitoring of MB-102 clearance has previously been demonstrated in rodents and dogs to be well correlated with plasma clearance kinetics. ${ }^{10}$ The purpose of the present work was to develop a device suitable for clinical testing in humans. The transdermally monitored terminal rate constant for clearance of MB-102 by the kidneys is expected to be directly predictive of patient GFR, normalized to body size. The ultimate aim of this work is to provide a transdermal monitor for GFR that is real-time, in the sense that multiple estimates of the GFR are provided after a single agent injection. An initial measurement on a human subject is presented here, but the focus 
of this paper is on the instrument design, verification, and validation to a sufficient level to warrant use in human clinical studies. The term "verification" is used in this paper to describe in vitro test procedures intended to determine whether the instrument is functioning as designed while "validation" is used to describe in vivo procedures designed to test whether the instrument accomplishes its intended purpose.

\section{Methods}

\subsection{Instrument Description}

Two identical instruments were constructed and tested, hereafter referred to as unit 1 and unit 2 . A schematic of the instrument is provided in Fig. 1(a).

(a)

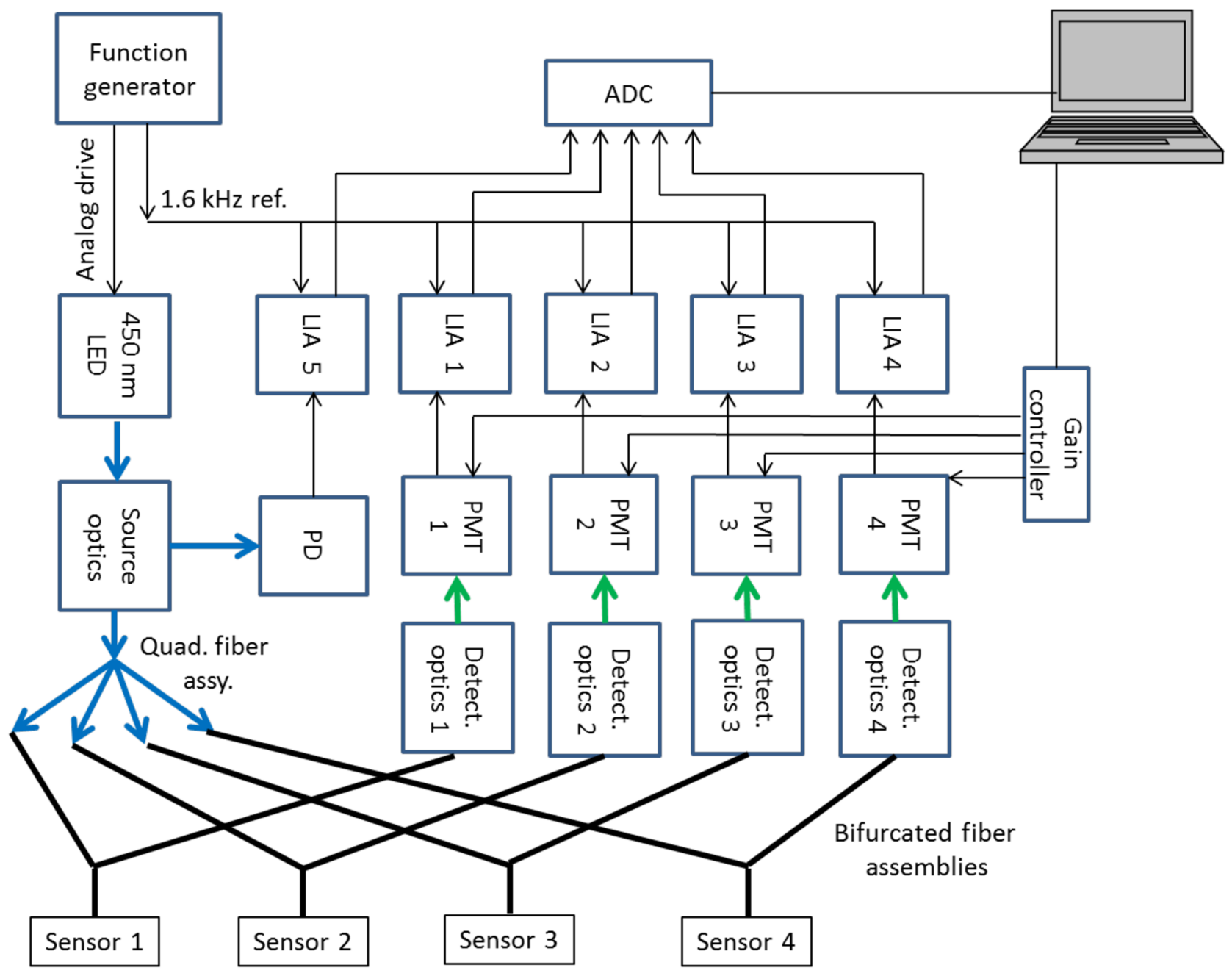

(b)
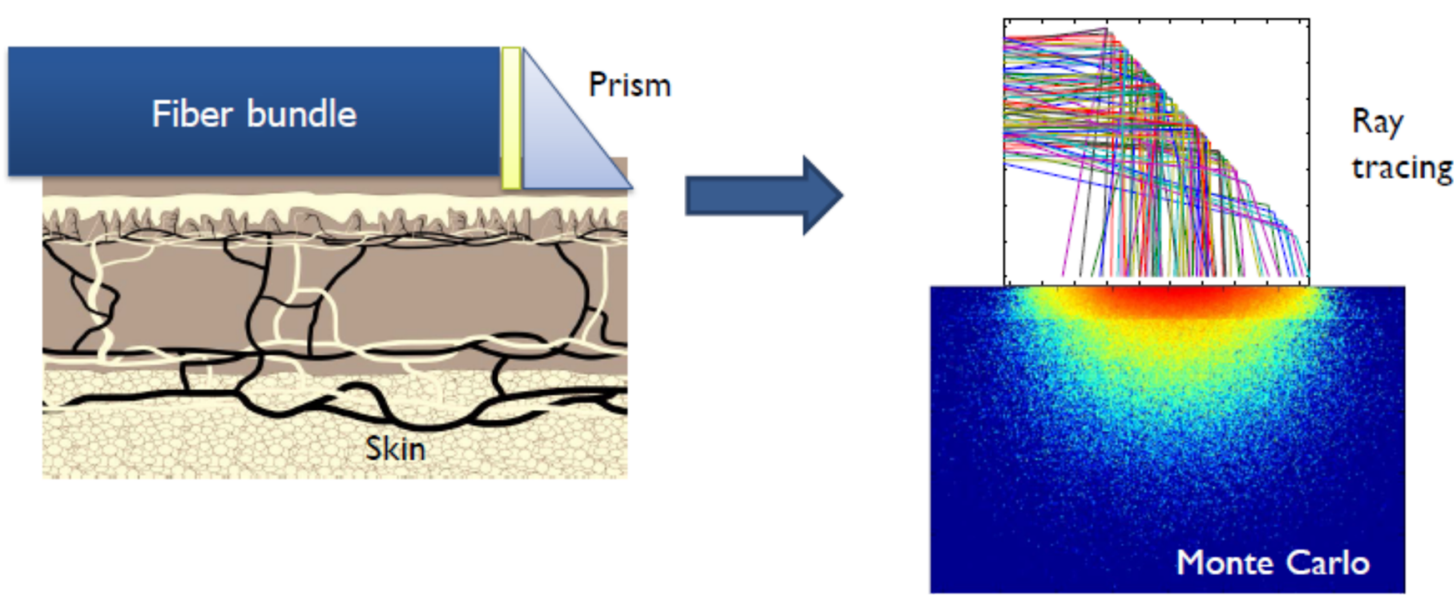

Fig. 1 (a) Instrument schematic. ADC, analog-to-digital converter; LIA, lock-in amplifier; PMT, photomultiplier tube. (b) Schematic of the numerical modeling of the sensor and skin. 
The light source was a 455-nm light-emitting diode (LED) (Lightspeed Technologies Inc., Model LEDHR-455) packaged in a driver with integrated optics (Lightspeed Technologies Inc., Model HPLS-36AD3500), which was collimated (Lightspeed Technologies Inc., Model $\mathrm{OH} 36-24 \times 11$ ) before passing through a 10-nm (full-width at half-maximum) bandpass filter centered at $450 \mathrm{~nm}$ (Omega Optical, Model 450BP10), and then focused (LightSpeed Technologies Inc., Model FA-Oriel$11 \mathrm{~mm}$ ) onto the common end of a quadfurcated fiber bundle (Newport Corp., Model SP1000-5413 Special). Each leg of the quadfurcated fiber assembly was then directly coupled into one branch of a bifurcated fiber bundle (Newport Corp., Model SP77533-5390 Special). The light level was controlled by adjusting the amplitude of a $1.6-\mathrm{kHz}$ square-wave modulated LED driver signal from a function generator (Agilent, Model 22522B) and by adjusting the air gap between the quadfurcated and bifurcated fiber bundle ends. The power level at the sample interface was set to $10 \pm 0.2 \mu \mathrm{W}$ for each sensor, using an optical power meter (Thorlabs Inc., Model S120C sensor with PM100USB meter). The fiber optic bundle consisted of a randomly mixed close-packed array of the source and detection fibers (individual fiber diameter: $55 \mu \mathrm{m}, 0.56 \mathrm{NA}$, common bundle diameter: $4.5 \mathrm{~mm}$, total bundle length: $2 \mathrm{~m}$ ). In some cases, a right angle prism (Edmund Optics, Model 47-594) was attached to the common end of the bifurcated fiber bundle using a UV-cured epoxy (Loctite, Model M-31CL), thereby making the fiber cable parallel to the skin surface and easier to secure. The irradiance at the sample was estimated to be 6.5 or $50 \mu \mathrm{W} / \mathrm{cm}^{2}$ with and without the prism, respectively; about 200 or 30 times lower than the power limit for photodecay of endogenous chromophores in human skin established previously. ${ }^{11}$

After injection of the source light into the sample, the same fiber bundle was used to collect the backscattered source and fluorescence light. The detection leg of each bifurcated fiber bundle was collimated (Newport Corp., Model 77645) before passing through a 100-nm (full-width at half-maximum) bandpass filter centered at $570 \mathrm{~nm}$ (Omega Optical, Model 570AF100), and detected by a photomultiplier tube (PMT) with built-in amplifier (Hamamatsu Photonics Co., Model H7827011). The PMT gain was digitally controlled through a customwritten LabVIEW-based (National Instruments, Version 2011, SP1) software interface. The amplified PMT output was synchronously detected by a miniature lock-in amplifier (Femto Messtechnik GmbH, Model LIA-MV-150-S) referenced to the frequency source that was used to drive the LED modulation. The analog output of the lock-in amplifiers was routed into a 16-bit analog-to-digital converter (National Instruments, Model NI USB-6218) before being read, displayed, and saved using a custom software interface.

The detector gain levels were adjusted to target between $30 \%$ and $75 \%$ saturation by using a $1-\mathrm{cm}$ glass cuvette containing $2-\mu \mathrm{M} \mathrm{MB}-102$, and then were fixed throughout each experiment. Data were read, displayed, and immediately saved by the software at a rate of $1 \mathrm{~Hz}$. The saved data streams included the raw fluorescence intensity, the detector gain levels, the monitoring signal, and the internal instrument temperature.

Fitting of the in vivo fluorescent data was performed in a custom-written LabView interface using nonlinear least-squares method of Levenberg-Marquardt. ${ }^{12,13}$ Prior to curve fitting, the initial baseline was subtracted, the time axis was adjusted so that the time of injection occurred at time 0 , and the peak

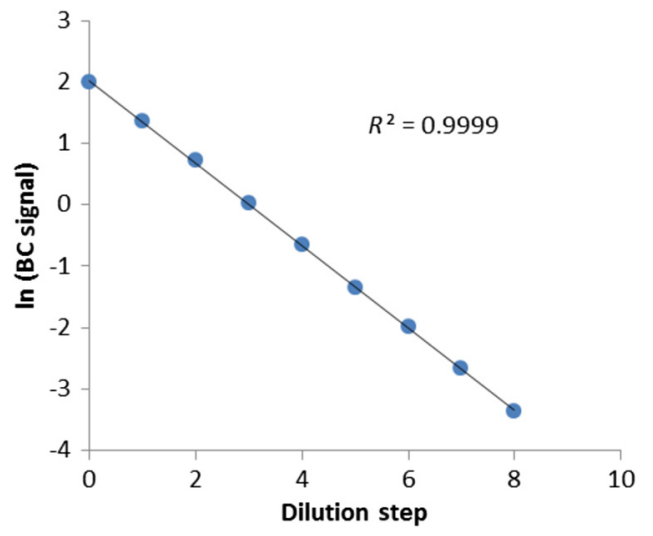

Fig. 2 Instrument testing using cuvettes: example graph (sensor 2, unit 1) showing a linear fit to the logarithm of background-corrected (BC) fluorescence intensity versus dilution step. The experimental data are depicted with blue circles. The fit is depicted with a black line.

amplitude was normalized to 1 . The resulting decay curves were fit to a single exponential between 30 and 120 min after agent injection. The offset value was fixed at zero.

A numerical model of the fiber bundle, prism, and human skin was developed, as shown in Fig. 1(b), and has been described elsewhere. ${ }^{14}$ Due to the small effective sourcedetector spacing, the model predicts that the average measurement depth is shallow, about $200 \mu \mathrm{m}$ below the skin surface. In human skin, this depth corresponds approximately to the shallow dermis, just below the superficial plexus of blood vessels at the junction between the epidermis and dermis. Due to the overlapping of the source and detector emission and detection cones within the prism, having a small gap between the prism and skin is expected to have little effect on the effective measurement depth in skin. For convenience, in the described rodent studies, the prism was held about 1 to $2 \mathrm{~mm}$ above the skin. For the human studies, the prism was attached to the surface of the skin using a medical grade two-sided adhesive (3M 1577).

\subsection{MB-102 Fluorescent Agent Properties}

The fluorescent agent, MB-102 (MediBeacon Inc.), is a small molecule (MW: $372.3 \mathrm{~g} / \mathrm{mole}$ ) belonging to the general class of compounds known as pyrazines. In phosphate buffer, the fluorescence emission peak at $557 \mathrm{~nm}$ is shifted by more than $100 \mathrm{~nm}$ from the absorbance peak $(435 \mathrm{~nm}) .{ }^{15}$ Prior studies in rats and dogs have shown that the agent is freely filtered by the kidneys, is not significantly metabolized, and has no observed detrimental effects at doses up to 200 to 300 times the estimated human dose. ${ }^{9}$ After intravenous bolus injection, pharmacokinetic studies have demonstrated that MB-102 distributes into the extracellular space before being cleared by the kidneys. ${ }^{9,15}$

\subsection{Clinical Requirements for GFR Measurement}

There is a strong medical need for improved monitoring of both chronic kidney disease (CKD) and acute kidney injury (AKI).

For monitoring AKI, such as during administration of potentially nephrotoxic agents, not just the absolute GFR, but its change on a time scale of hours to days would be of diagnostic value for clinicians. In the absence of real-time GFR monitoring capability, AKI is currently staged on the basis of serum creatinine and urine output, as summarized in columns 1 to 3 of 
Table 1 Stages of AKI and predicted GFR change. Columns 1 to 3 are adapted from Table 2 of Ref. 16. Columns 4 and 5 were derived from serum creatine concentrations in column 2, using the 2009 CKD-EPI creatine equations. ${ }^{17}$

\begin{tabular}{|c|c|c|c|c|}
\hline AKI stage & Serum creatinine & Urine output & $\begin{array}{c}\text { Predicted GFR } \\
\text { change, female (\%) }\end{array}$ & $\begin{array}{c}\text { Predicted GFR } \\
\text { change, male (\%) }\end{array}$ \\
\hline 1 & $\begin{array}{l}1.5 \text { to } 1.9 \text { times baseline or } \\
\geq 0.3 \mathrm{mg} / \mathrm{dl} \text { increase }\end{array}$ & $<0.5 \mathrm{ml} / \mathrm{kg} / \mathrm{h}$ for 6 to $12 \mathrm{~h}$ & $12 \%$ to $20 \%$ & $15 \%$ to $25 \%$ \\
\hline 2 & 2.0 to 2.9 times baseline & $<0.5 \mathrm{ml} / \mathrm{kg} / \mathrm{h}$ for $12 \mathrm{~h}$ & $20 \%$ to $30 \%$ & $25 \%$ to $36 \%$ \\
\hline 3 & $\begin{array}{l}3.0 \text { times baseline or increase in creatinine to } \\
\geq 4.0 \mathrm{mg} / \mathrm{dl} \text { or initiation of renal replacement } \\
\text { therapy or in patients }<18 \text { years, decrease in } \\
\text { eGFR to }<35 \mathrm{ml} / \mathrm{min} / 1.73 \mathrm{~m}^{2}\end{array}$ & $\begin{array}{c}<0.5 \mathrm{ml} / \mathrm{kg} / \mathrm{h} \text { for } 24 \mathrm{~h} \text { or } \\
\text { anuria for } \geq 12 \mathrm{~h}\end{array}$ & $\geq 30 \%$ & $\geq 36 \%$ \\
\hline
\end{tabular}

Table 1, adapted from the 2012 KDIGO guidelines for AKI. ${ }^{16}$ The importance of AKI staging is as a guide to treatment, with, for example, discontinuation of nephrotoxic agents recommended if possible at AKI stage 1, and consideration of renal replacement therapy at AKI stage $2 .{ }^{16}$ To predict the expected change in GFR associated with AKI, the serum creatine values at each AKI stage in Table 1 were converted into GFR values, using the 2009 CKD-EPI creatinine equation. ${ }^{17}$ The CKD-EPI equations are dependent on age, gender, and ethnicity, so these factors, along with different baseline creatinine levels, corresponding to different starting stages of CKD, were included in the modeling. It was found that only gender has an effect of the relative change in GFR associated with AKI stages, as summarized in the final 2 columns of Table 1 . These results suggest that, assuming a normal distribution of errors in GFR prediction, in order to avoid misdiagnosis of AKI stage by more than 1 stage with $95 \%$ confidence, the standard relative error for GFR prediction (i.e., measurement precision) will need to be about $5 \%$ or less. For this reason, for the purposes of instrument performance verification, an accuracy target of 5\% was selected.

The length of time over which GFR monitoring can take place after a single administration of the MB-102 agent will vary according to the kidney health of the patient. For patients with normal kidney function, the time constant for agent clearance is expected to be on the order of $2 \mathrm{~h}$, based on prior animal studies, and based on the clearance times observed for other GFR agents (e.g., iohexol, Tc-DTPA) that distribute across the extracellular fluid space. ${ }^{18}$ Monitoring of the renal clearance of the MB-102 agent is expected to last for several time constants, so the initial study on healthy human subjects monitoring was planned for about $6 \mathrm{~h}$ following agent injection. For patients with compromised kidney function, it is anticipated that MB-102 tracer monitoring after a single agent administration could last for as long as $24 \mathrm{~h}$ or more.

\subsection{In Vitro Device Verification}

The ultimate purpose of the prototype instrument is to provide an accurate transdermal measurement of the rate of renal clearance of the fluorescent agent. After injection and initial equilibration into the body, the logarithm of the fluorescence intensity of the agent, $I$, is expected to decay linearly with time, $t$, as shown in Eq. (2) (assuming equilibration rate $\left.\gg k_{r}\right)^{19}$

$I=I_{0} e^{-k_{r} t}$, $\ln (I)=-k_{r} t+\ln \left(I_{0}\right)$.

The slope of this line, $k_{r}$, is the renal clearance rate (in units of, for example, $1 / \mathrm{min}$ ). To assess the overall system performance of the prototype instruments, a series of cuvettes was prepared with descending MB-102 concentrations, to mimic the decay observed in the human body due to renal clearance. A scattering agent, $1 \%$ Intralipid (Sigma-Aldrich, CAS Number 68890-65-3), was added to all cuvettes to mimic the scattering properties of human skin in the visible range. Prior work has determined that the reduced scattering coefficient of human skin and $1 \%$ Intralipid fall in about the same range $(\sim 0.5$ to $3 \mathrm{~mm}^{-1}$ ) for visible light. ${ }^{20-22}$ The agent concentrations were prepared in a geometric series, with the series factor being 2 . The highest and lowest concentrations were 8 and $0.03125 \mu \mathrm{M}$, respectively. There were therefore nine solutions in the dilution series, plus a 10th solution containing no fluorescent agent: $8,4,2 \ldots 0.03125,0 \mu \mathrm{M}$. The highest concentration was chosen to correspond twice the expected peak tissue concentration of the fluorescent agent, based on the planned injected dose for the human study, and the expected volume of distribution (i.e., the extracellular space) in a typical human subject. The solution containing no fluorescent agent was used as a baseline and was subtracted from all other measurements.

Analogous to the in vivo renal clearance of the fluorescent agent, the logarithm of the fluorescence intensity of the scattering cuvettes, as a function of dilution step, $d$, was expected to decay linearly, as shown in Eq. (4)

$$
\begin{aligned}
& I=I_{0} e^{-f d}, \\
& \ln (I)=-f d+\ln \left(I_{0}\right), \\
& d=\ln (2) .
\end{aligned}
$$

For a geometric series with series factor 2, the expected slope of the line, $d$, is $\ln (2)$, as shown in Eq. (5). A standard error in the slope estimate of less than $5 \%$ was chosen as the verification criterion. Error in the estimation of the slope will translate directly into error in GFR estimation.

The probe configuration for the cuvette measurements was the same as that used for the in vivo experiments, except the probe and cuvette were held within a fixture that angled the optical axis of the light at an angle of $30 \mathrm{deg}$ from normal incidence, in order to minimize reflections from the faces of the cuvette. 


\subsection{In Vivo Device Validation}

All animal testing was performed using Sprague-Dawley male rats. The fluorescent agent was injected at a concentration of $2 \mathrm{mM}$ in PBS, followed by a flush with $0.25 \mathrm{~mL}$ of sterile saline.

Unit 1 was tested on 12 rats at Saint Louis University (St. Louis, Missouri) under an IACUC-approved protocol. The animal weights were between 320 and $370 \mathrm{~g}$. Anesthesia was performed by injection of $124 \mathrm{mg} / \mathrm{kg}$ ketamine and $12.5 \mathrm{mg} / \mathrm{kg}$ xylazine. The initial dose was $1 \mathrm{~mL} / \mathrm{kg}$ animal weight. Additional injections were made abdominally, as necessary to prevent the animals from waking up. The MB-102 fluorescent agent was injected into a central vein. In the nine animals, the injected volume was $1.0 \mathrm{~mL}$ while in the remaining three animals the injected volume was $0.75 \mathrm{~mL}$.

Instrument unit 2 was tested on nine animals at Washington University in St. Louis (St. Louis, Missouri) under an IACUCapproved protocol. The animal weights were between 250 and $275 \mathrm{~g}$. Isoflurane was used for anesthesia at $1.5 \%$ and $2 \%$ levels. The fluorescent agent was injected into the tail vein. The injected volume was $1.0 \mathrm{~mL}$ in all nine animals.

\section{Results and Discussion}

\subsection{In Vitro Verification Testing}

The stability of the instruments over a 6-h time period (corresponding to the time period over which subjects were monitored in the clinical study) under normal laboratory conditions was tested by placing cuvettes containing $0.3 \mu \mathrm{M}$ MB-102 in front of two sensors on each instrument. After normalizing by the LED monitoring signal, the drift of the signal over time, computed as the median absolute deviation divided by the median, was found to be less than $0.5 \%$ in all cases. The root mean squared (RMS) noise of the instruments was estimated from $60-\mathrm{s}$ measurements on a $1-\mathrm{cm}$ path length cuvette containing $4 \mu \mathrm{M}$ MB-102. The RMS noise, defined as the ratio of the standard deviation to the average across the 60 measurements, was less than $0.1 \%$ for both instruments. Cross-talk between sensor channels was explored by comparing measurements with one sensor on a $1-\mathrm{cm}$ path length cuvette containing no fluorescent agent (PBS only) while placing cuvettes filled with either PBS only or $10 \mu \mathrm{M}$ MB-102 in PBS in front of all of the remaining sensors. The cross-talk effect was less than $0.1 \%$ across all channels on both instruments.

Linearity testing using a dilution series of cuvettes was performed on all four probe channels on both of the two prototype instruments. An example plot is shown in Fig. 2. The disagreement between the fitted and expected slope was less than $4 \%$ for all four channels on both instruments, as summarized in Table 2. The coefficient of variation of the slope estimation across the four different sensors on each instrument was less than $3 \%$. Subsequent retesting over time and on different batches of prepared cuvettes pointed to errors in solution preparation and solution aging as the principal sources of error in the slope estimation.

\subsection{Background Signal in Cuvettes}

In the absence of the MB-102 agent, a background signal was observed on cuvettes filled with only $1 \%$ Intralipid. This background signal was about $5 \%$ of the signal measured on a cuvette containing $4 \mu \mathrm{M}$ MB-102 in addition to $1 \%$ Intralipid. Several experiments were performed in order to explore the origins of
Table 2 Summary of system verification with scattering cuvettes containing a scattering agent and a dilution series of MB-102. The theoretically expected value for $d$, is the log of the dilution factor, or $\ln (2)$. The error is the difference between the least-squares fitted slope of the natural logarithm of the fluorescence intensity versus the dilution step number. The pass/fail (P/F) threshold was $5 \%$ error.

\begin{tabular}{|c|c|c|c|c|c|c|c|}
\hline \multirow[b]{2}{*}{ Sensor } & \multirow{2}{*}{$\frac{\text { Expected }}{D}$} & \multicolumn{3}{|c|}{ Unit 1 (measured) } & \multicolumn{3}{|c|}{ Unit 2 (measured) } \\
\hline & & $d$ & Error & $\mathrm{P} / \mathrm{F}$ & $d$ & Error & $\mathrm{P} / \mathrm{F}$ \\
\hline 1 & 0.6931 & 0.6732 & $-2.9 \%$ & Pass & 0.7039 & $1.6 \%$ & Pass \\
\hline 2 & 0.6931 & 0.6714 & $-3.1 \%$ & Pass & 0.7086 & $2.2 \%$ & Pass \\
\hline 3 & 0.6931 & 0.6720 & $-3.1 \%$ & Pass & 0.7055 & $1.8 \%$ & Pass \\
\hline 4 & 0.6931 & 0.6715 & $-3.1 \%$ & Pass & 0.7063 & $1.9 \%$ & Pass \\
\hline
\end{tabular}

this background signal. In some experiments, the PMT detector was replaced with a spectrometer (Ocean Optics, USB2000). The spectrum of the agent-free cuvette, shown in Fig. 3, decreased monotonically with increasing wavelength. Decreasing the optical fiber bundle length from 2 to $1 \mathrm{~m}$ decreased the background signal by about $6 \%$. Replacing the $1-\mathrm{m}$ glass fiber bundle with a fused silica fiber bundle (Newport Corp., Model 77565) further decreased the background signal, by about another $6 \%$. Fluorescence from Intralipid itself was found to be negligible by varying the Intralipid concentration, and normalizing the agent-free signals using separate cuvettes spiked with MB-102 agent in addition to the Intralipid. The background signal was found to depend strongly on the position of the collimating lenses used in front of the bandpass filters in both the source and detection assemblies. It was concluded from these experiments that the 2-m glass fiber used in the standard configuration of the instrument contributes a background fluorescence signal that is about $12 \%$ of the background signal observed in a $1-\mathrm{cm}$ cuvette filled with $1 \%$ Intralipid. The remaining background signal appears to be due to imperfect rejection of source light through the optical filters.

\subsection{Cuvette Depth Dependence}

To better understand the range of depths from which the fluorescence is being measured, one of the instruments (unit 2) was

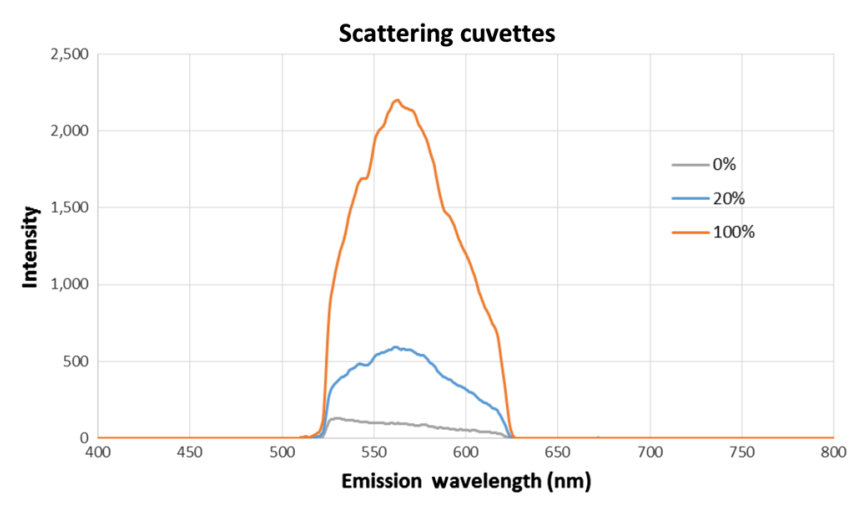

Fig. 3 Spectra of a $1-\mathrm{cm}$ path length cuvette filled with $1 \%$ Intralipid and variable concentrations of MB- $102(100 \%=4 \mu \mathrm{M})$. The apparatus used to collect the spectra was the same as in Fig. 1(a), except the PMT detector was replaced with a spectrometer. 


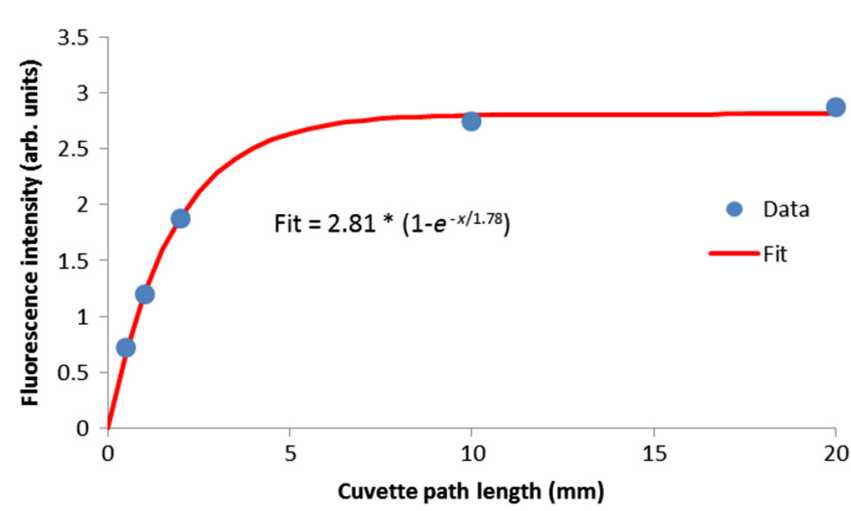

Fig. 4 Measurement of 0.2- $\mu \mathrm{M}$ MB-102 fluorescence in 1\% Intralipid as a function of cuvette path length.

used to measure fluorescence in cuvettes having several different path lengths, using the same sensor backscattering geometry as employed for in vivo measurements (Fig. 4). All of the cuvettes contained $1 \%$ Intralipid and $0.2 \mu \mathrm{M}$ MB-102. The nearly identical fluorescence intensity that was measured with the 10 and $20 \mathrm{~mm}$ pathlength cuvettes indicates that there is little measurement sensitivity to photons traveling deeper than $10 \mathrm{~mm}$. The observed depth-dependence was well described by an asymptotic exponential model with a fitted exponential constant of $1.8 \mathrm{~mm}$. Monte Carlo modeling of this experiment was in close agreement with these results, further details of which have been described elsewhere. ${ }^{14}$

\subsection{In Vivo Validation Testing}

A total of 21 anesthetized rats were tested on the two different prototype instruments. The body sites selected for transdermal measurement were the ear, ankle, and tail. The ear was measured on all animals. In addition, the ankle was measured on two animals and the tail on one. The ear was glued to a glass slide using cyanoacrylate, whereas the ankle and tail sites were unsecured. Each fiber sensor was held in a fixed position about 1 to $2 \mathrm{~mm}$ above the skin. In three of the measurements, the peak signal after MB-102 injection was overloaded for several minutes, preventing estimation of the peak height.

Example time course plots are shown in Figs. 5 and 6. The results of fitting the terminal fluorescence decay constant $\left(\tau_{r}\right)$ on

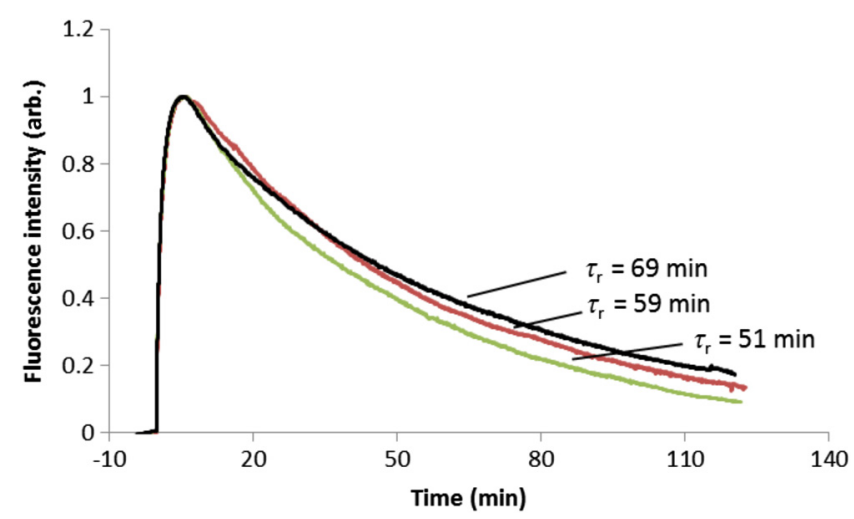

Fig. 5 Example time course plots of MB-102 injection, distribution, and clearance, as measured on the ear of the different rats. The fluorescence intensities have been offset and normalized to one. Time zero corresponds to the time of injection for each animal.

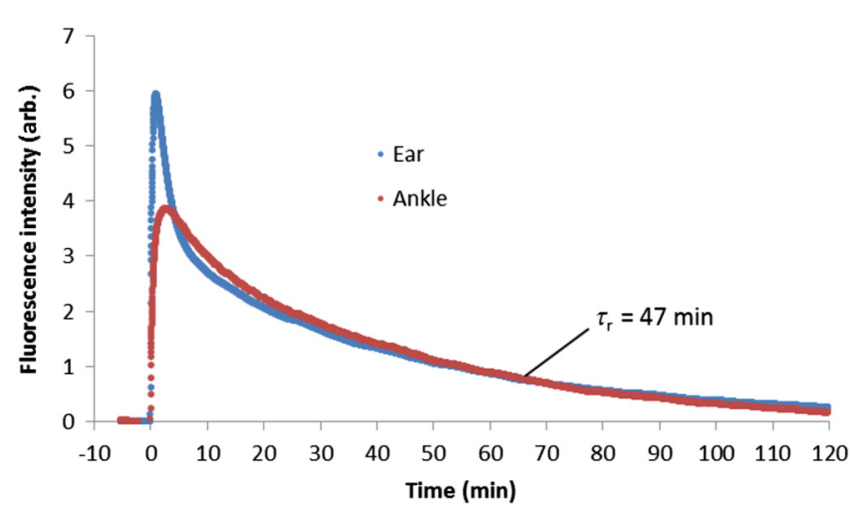

Fig. 6 Example time course plots of MB-102 injection, distribution, and clearance, as measured on the ear and ankle of the same rat. The fluorescence intensities have been baseline offset but not normalized.

the ear are summarized in Table 3. Substantial variability was observed among the different animals. Table 3 shows the mean and standard deviation of the decay constants across all animals and across subgroups with different anesthesia methodologies. The animals treated with ketamine/xylazine showed wider variability (standard deviation: $13 \mathrm{~min}$ ) than those treated with $2 \%$ isoflurane (standard deviation: $7 \mathrm{~min}$ ). Decreasing the isoflurane concentration from $2 \%$ to $1.5 \%$ resulted in a decrease in the mean decay constant, but additional measurements would be needed to determine whether this decrease was statistically significant.

To test whether the variation in the renal decay constant was related to the specific body site of the measurement or to the specific channel of the instrument, in three cases more than one probe was attached to different body sites on the same animal (see Fig. 6 for example time course plots). The within-animal variation of the decay constant was computed by combining the individual-animal standard deviations as a root-mean-square across all animals on which multiple probes were employed. The between-animal variation was computed by combining the standard deviations for each group of four simultaneously measured animals, as a root-mean-square across all groups. As shown in Table 4, the low within-animal variation (1 min) compared to the between-animal variation $(14 \mathrm{~min})$ points to animal-to-animal physiological variation as the primary cause of the decay constant variation.

Although the terminal fluorescence decay was consistent across different body sites on the same animal, the initial rise

Table 3 Averaged terminal decay constants for different anesthetic treatments (ear body site). The number of animals used to compute the mean and standard deviation is displayed in the column labeled $N$.

\begin{tabular}{lccc} 
Anesthesia & $N$ & $\begin{array}{c}\text { Mean } \\
(\mathrm{min})\end{array}$ & $\begin{array}{r}\text { Standard } \\
\text { dev. (min) }\end{array}$ \\
\hline All types & 19 & 46 & 11 \\
$\begin{array}{l}\text { Ketamine/ } \\
\text { xylazine }\end{array}$ & 12 & 44 & 13 \\
$\begin{array}{l}\text { 2\% isoflurane } \\
1.5 \% \text { isoflurane }\end{array}$ & 5 & 54 & 7 \\
\hline
\end{tabular}


Table 4 Standard deviation of terminal decay constants between different animals versus between different body sites on the same animal.

\begin{tabular}{lcc}
\hline Grouping & $\begin{array}{c}\text { Number of } \\
\text { animals }\end{array}$ & $\begin{array}{c}\text { Standard } \\
\text { deviation (min) }\end{array}$ \\
\hline $\begin{array}{l}\text { Within-animal: different body sites } \\
\text { on the same animal }\end{array}$ & 3 & 1 \\
$\begin{array}{l}\text { Between-animal: same body } \\
\text { site (ear) across multiple animals }\end{array}$ & 20 & 14 \\
\hline
\end{tabular}

of the fluorescence varied substantially between body sites. For example (see Fig. 6), when sensors were placed on the ear and ankle of the same animal, the fluorescence measured on the ear peaked more rapidly and to a higher level than that on the ankle, before leveling out to have a well-matched terminal decay. This behavior is consistent with a two-compartment model, in which after injection, the agent requires several minutes to equilibrate between the vascular space and the rest of the extracellular space. According to this paradigm, the spot measured on the ear was more highly vascularized than was the ankle.

The baseline and peak signal sizes and their ratio are summarized for the different body sites in Table 5. Both the ankle and tail sites showed higher baseline fluorescence than the ear site. The tail is known to have high collagen content, one of the principal sources of intrinsic fluorescence in the spectral region where the instrument is measuring (excitation: $450 \mathrm{~nm}$, emission: $570 \mathrm{~mm}){ }^{23}$

Estimates of the in vivo signal-to-noise ratio (SNR) were made for three of the transdermal ear measurements, as shown in Fig. 7. The root mean square of the residual from single exponential fits between 30 and 100 min after MB-102 injection was used as the estimate of noise, whereas the mean of the fluorescence signal over this time period was used as the signal estimate. The SNR ranged between 50 and 150 for the three animals. The larger amplitude of the fitting residual in the 30- to 40-min region for two of the three animals suggests that the distribution (or equilibration) of the agent may not yet be complete at $30 \mathrm{~min}$, and that improvement in the fitting precision might be realized by waiting until $40 \mathrm{~min}$ to start applying the single exponential fitting. For the animal with the highest SNR, the self-consistency of the fitted renal decay rate error was estimated using different fitting window time lengths between 5 and $20 \mathrm{~min}$, with multiple windows positioned sequentially (with no overlap) between 30 and $100 \mathrm{~min}$. With a fitting window size of $10 \mathrm{~min}$, the precision of the decay rate estimate across the different fitting windows was $5.2 \%$. The relationship between the fitting window time length and the precision of the decay rate estimate is summarized in Fig. 8.
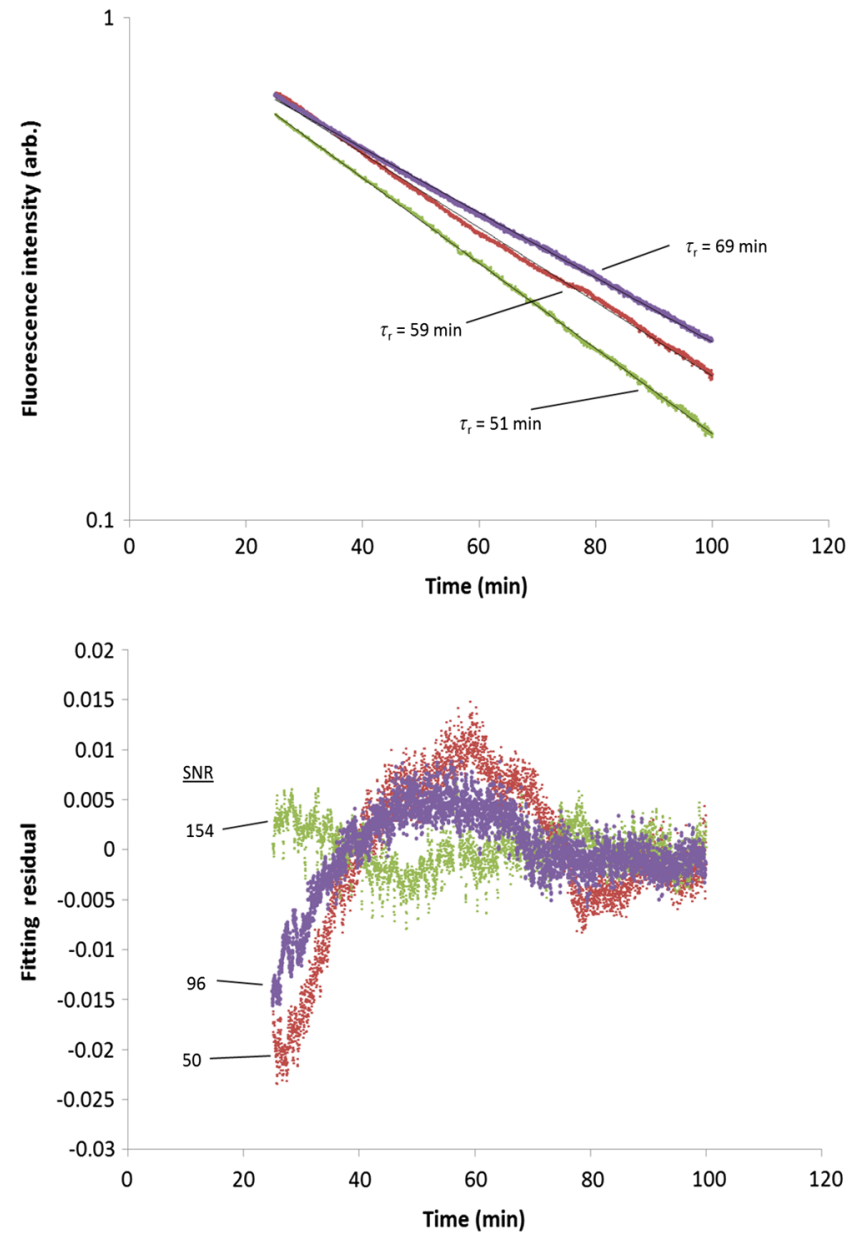

Fig. 7 (a) Semilog plots of single exponential fits to transdermal fluorescence decay on the ear of three different rats between 30 and 100 min after MB-102 injection, and (b) estimate of SNR from the residual of single exponential fits.

\subsection{Initial Clinical Trial Results}

A clinical trial was performed on 16 healthy subjects having a range of skin color types, with the aim of demonstrating the feasibility of transdermally monitoring renal clearance of the MB-102 fluorescent tracer agent. The University of Maryland Medical School IRB approved the protocol and study. The bolus injection of MB-102 administered by intravascular injection on each subject was varied between 0.5 and $4 \mu \mathrm{mol} / \mathrm{kg}$ subject weight. Sensors placed at four different body sites (sternum, forehead, arm, and side) on each subject provided simultaneous measurements of the MB-102 fluorescent time course. An example fluorescence time-profile for a sensor placed on the

Table 5 Relative signal levels at baseline and peak on different body sites. The baseline is the fluorescence signal observed prior to administration of the MB-102 agent. The peak is the maximum fluorescence signal observed after agent injection.

\begin{tabular}{|c|c|c|c|c|c|c|c|}
\hline Body site & Number of animals & Baseline & Standard deviation & Peak & Standard deviation & Peak/baseline & Standard deviation \\
\hline Ear & 19 & 1.3 & 0.3 & 9.3 & 1.5 & 7.5 & 1.7 \\
\hline Ankle & 1 & 4.2 & & 10.7 & & 2.5 & \\
\hline Tail & 1 & 5.3 & & 6.4 & & 1.2 & \\
\hline
\end{tabular}




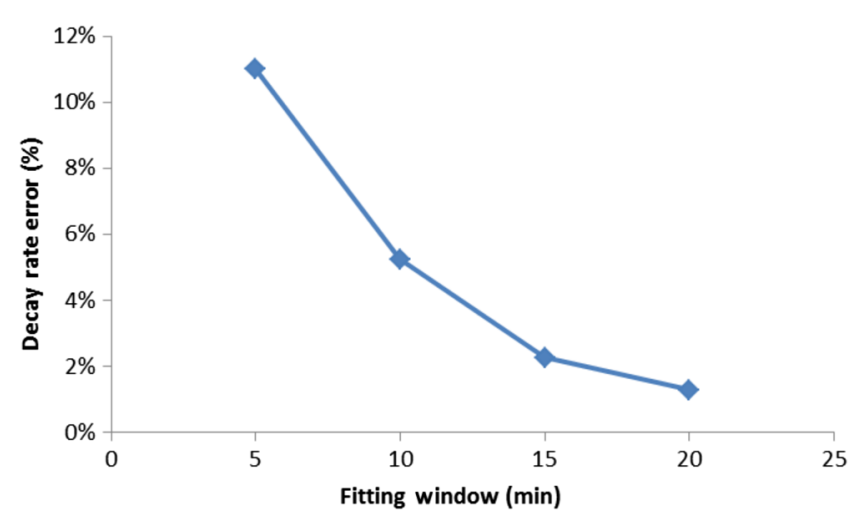

Fig. 8 Relationship between single exponential decay rate fitting error and window size, from the transdermal fluorescence monitoring on the ear of one animal.

sternum of a subject with Fitzpatrick skin types 3 and 4, administered with $4 \mu \mathrm{mol} / \mathrm{kg} \mathrm{MB}-102$, is shown in Fig. 9. A twoexponential fit (solid red line) is shown overlaid with the fluorescence measurements (blue dots). The data points were smoothed by averaging the individual measurements (collected at about once per second) across a 40-s window. The time constant of the terminal exponential decay term was $\sim 127 \mathrm{~min}$. A full report on the clinical trial results will be provided elsewhere.

In future studies, including subjects with compromised renal function, the aim will be able to relate this time constant to GFR (indexed to body size). The precedent for directly relating a transdermally measured renal clearance of an exogenous agent directly to GFR (indexed to body surface area) has been established in previous studies employing a radioactive tracer agent, Tc-DTPA. ${ }^{18}$ In those studies, a strong linear correlation $\left(R^{2}=0.97\right)$ was observed across a pool of 50 subjects having a wide range of GFR. Like MB-102, Tc-DTPC is a distributed across the extracellular space of the body. A similar relationship between the terminal decay kinetics and GFR is therefore expected for the MB-102 agent.

By applying the slope determined by Rabito et al. ${ }^{18}$ for TcDTPA to the decay constant extracted from the example subject displayed in Fig. 9 that was administered MB-102, the predicted GFR is $130 \mathrm{~mL} / \mathrm{min} / 1.73 \mathrm{~m}^{2}$. This value is indicative of healthy kidney function, as expected (subjects were screened for healthy kidney function during enrollment). The determination of the specific slope applicable to the MB-102 agent will be an important goal of future clinical studies. But by applying the slope from Rabito et al., ${ }^{18}$ a rough prediction of the decay time constants expected for subjects at progressively worsening stages of CKD from stages 1 to 4 , results in 160 (stage 1), 220 (stage 2), 360 (stage 3), and $700 \mathrm{~min}$ (stage 4).

It is important to note that the measured rate constant $\left(k_{r}\right)$ is inherently proportional to GFR normalized (or in clinical parlance "indexed") to the volume of distribution $\left(V_{d}\right),{ }^{24}$ as

$k_{r}=\frac{1}{\tau_{r}}=\frac{\mathrm{GFR}}{V_{d}}$.

The high correlation coefficient between $k_{r}$ and BSAindexed GFR that was observed by Rabito et al. ${ }^{18}$ suggests that BSA-indexed GFR, which is the standard method of reporting GFR in the clinic is substantially equivalent to indexing by volume of distribution. The topic of the most appropriate method of indexing GFR has been a matter of some debate in the clinical community, ${ }^{25-27}$ and may be worth further exploration once sufficient clinical data have been collected to test the different proposed indexing methods.

\section{Summary and Conclusions}

Two prototype instruments constructed for the purpose of monitoring up to four body sites simultaneously in human trials were tested both with cuvettes filled with varying concentration of MB-102 and Intralipid, and on 21 rats injected with MB-102 and monitored transdermally. The cuvette tests showed the instruments to have low short term ( $1 \mathrm{~min}) \mathrm{RMS}$ noise $(<0.1 \%)$, low cross-talk between channels $(<0.1 \%)$, and low signal drift $(<0.5 \%$ over $6 \mathrm{~h}$, under normal laboratory conditions). A dilution series of cuvettes was used to demonstrate better than 5\% accuracy in determining the rate constant for fluorescent decay with increasing dilution level.

In the animal experiments, a wide variation in the terminal fluorescence decay constant was observed across animals (standard deviation: $11 \mathrm{~min}$ ). At least part of this variability appeared to be related to the type or concentration of the anesthetic agent. Measurements of the same animal at different body sites showed close agreement in the terminal decay time constant (standard deviation: $1 \mathrm{~min}$ ), despite initial differences in the fluorescence rise time. The variation in the initial kinetics may be explained by different local blood content of the body sites. In the best circumstances, the instrument SNR was $\sim 150$, allowing the renal clearance rate for MB-102 to be estimated with $5 \%$ precision using only a 10 -min data window. Scaling this window size according to the decay constant measured in rats ( $\sim 5 \mathrm{~min})$ compared to that anticipated in humans (about $2 \mathrm{~h}$ ) results in prediction of a 30-min fitting time window in humans. The motion noise associated with performing the transdermal

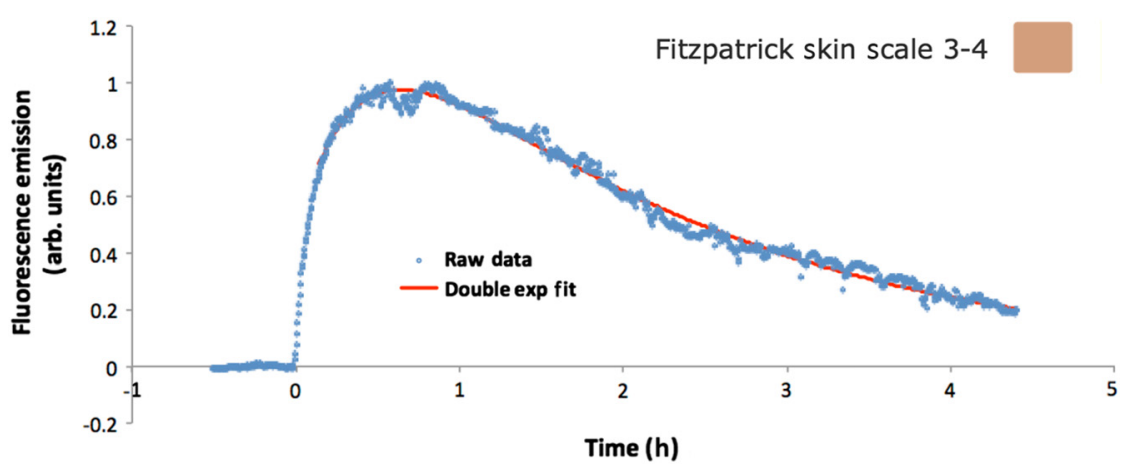

Fig. 9 Transdermal fluorescence from MB-102 measured at the sternum of a human subject. 
measurements on a fully conscious human, as compared to an anesthetized rat, will likely necessitate a further increase in window size in order to maintain a GFR precision of 5\%. This level of GFR precision is anticipated to be adequate to prevent misdiagnosis of AKI by more than one stage.

Based on these results, it was concluded that the instrument performance is sufficient to proceed to human trials. This instrument has subsequently been used in a FDA-approved pilot human clinical study on 16 healthy subjects. ${ }^{12}$ The aim of these studies is to establish whether the measurement accuracy and precision is sufficient to warrant proceeding with further studies on patients with renal disease, in which transdermal measurements of GFR will be compared with GFR determined by standard plasma pharmacokinetic analysis. The end goal of the commercial instrument is to provide bedside assessment in near real-time assessment of patient kidney function.

\section{Disclosures}

MediBeacon Inc. owns patents related to the described technology and supported this work financially. Both authors report owning equity interest in MediBeacon Inc.

\section{Acknowledgments}

The instrument construction and a subset of the verification testing were performed at RBC Medical Innovations by Richard Valenta and John Ellenz. The experiments at Saint Louis University were performed with the assistance of Stephen Rapp and his team at Seventh Wave Laboratories. The experiments at Washington University were performed with assistance from Dr. Michael Talcott and his staff at the Department of Comparative Medicine. The clinical study was conducted with investigators Thomas C. Dowling, PhD and Jeffrey C. Fink, MD, and personnel of the General Clinical Research Center, all at the University of Maryland, Baltimore.

\section{References}

1. L. A. Inker et al., "Estimating glomerular filtration rate from serum creatinine and cystatin C," N. Engl. J. Med. 367(1), 20-29 (2012).

2. M. A. Ferguson and S. S. Waikar, "Established and emerging markers of kidney function," Clin. Chem. 58(4), 680-689 (2012).

3. R. A. Star, "Treatment of acute renal failure," Kidney Int. 54, 1817-1831 (1998).

4. Z. H. Endre, J. W. Pickering, and R. J. Walker, "Clearance and beyond: the complementary roles of GFR measurement and injury biomarkers in acute kidney injury (AKI)," Am. J. Physiol. Renal Physiol. 301(4), F697-F707 (2011).

5. C. A. Rabito et al., "Optical, real-time monitoring of the glomerular filtration rate," Appl. Opt. 44(28), 5956-5965 (2005).

6. L. K. Chinen et al., "Fluorescence-enhanced europium-diethylenetriaminepentaacetic (DTPA)-monoamide complexes for the assessment of renal function," J. Med. Chem. 51(4), 957-962 (2008).

7. W. Yu, R. M. Sandoval, and B. A. Molitoris, "Rapid determination of renal filtration function using an optical ratiometric imaging approach," Am. J. Physiol. Renal Physiol. 292(6), F1873-F1880 (2007).

8. D. Schock-Kusch et al., "Transcutaneous measurement of glomerular filtration rate using FITC-sinistrin in rats," Nephrol. Dial. Transplant. 24(10), 2997-3001 (2009).

9. J. E. Bugaj and R. B. Dorshow, "Pre-clinical toxicity evaluation of MB102, a novel fluorescent tracer agent for real-time measurement of glomerular filtration rate," Regul. Toxicol. Pharmacol. 72(1), 26-38 (2015).

10. R. B. Dorshow et al., "Real-time point-of-care measurement of impaired renal function in a rat acute injury model employing exogenous fluorescent tracer agents," Proc. SPIE 8596, $85960 Z$ (2013).

11. M. P. Debreczeny et al., "Human skin auto-fluorescence decay as a function of irradiance and skin type," Proc. SPIE 7897, 78971T (2011).

12. K. Levenberg, "A method for the solution of certain non-linear problems in least squares," Q. Appl. Math. 2, 164-168 (1944).

13. D. Marquardt, "An algorithm for least-squares estimation of nonlinear parameters," SIAM J. Appl. Math. 11(2), 431-441 (1963).

14. K. M. Shultz et al., "Modeling of transdermal fluorescence measurements from first-in-human clinical trials for renal function determination using fluorescent tracer agent MB-102," Proc. SPIE 10079, 100790I (2017).

15. R. Rajagopalan et al., "Hydrophilic pyrazine dyes as exogenous fluorescent tracer agents for real-time point-of-care measurement of glomerular filtration rate," J. Med. Chem. 54(14), 5048-5058 (2011).

16. Eckard K. U. and B. L. Kasiske, http://kdigo.org/wp-content/uploads/ 2016/10/KDIGO-2012-AKI-Guideline-English.pdf (25 March 2018).

17. B. L. Kasiske and Wheeler D. C., http://kdigo.org/wp-content/uploads/ 2017/02/KDIGO_2012_CKD_GL.pdf (25 March 2018).

18. C. Rabito et al., "Accurate, fast, and convenient measurement of glomerular filtration rate in potential renal transplant donors," Transplantation 90(5), 510-517 (2010).

19. M. P. Debreczeny and R. B. Dorshow, "Development and clinical trial results of a prototype device for trans-cutaneous monitoring of kidney function," Proc. SPIE 10079, 100790K (2017).

20. G. Zonios and A. Dimou, "Light scattering spectroscopy of human skin in vivo," Opt. Express 17(3), 1256-1267 (2009).

21. S. T. Flock et al., "Optical properties of Intralipid: a phantom medium for light propagation studies," Lasers Surg. Med. 12, 510-519, 1992.

22. H. J. van Staveren et al., "Light scattering in Intralipid-10\% in the wavelength range of 400-1100 nanometers," Appl. Opt. 30, 4507-4514 (1991).

23. R. Gillies et al., "Fluorescence excitation spectroscopy provides information about human skin in vivo," J. Invest. Dermatol. 115(4), 704-707 (2000).

24. F. Keller, B. Schröppel, and U. Ludwig, "Pharmacokinetic and pharmacodynamic considerations of antimicrobial drug therapy in cancer patients with kidney dysfunction," World J. Nephrol. 4(3), 330-344 (2015).

25. F. W. Visser et al., "Feasibility and impact of the measurement of extracellular fluid volume simultaneous with GFR by 125I-iothalamate," Clin. J. Soc. Nephrol. 3, 1308-1315 (2008).

26. L. Hoste and H. Pottel, "Is body surface area the appropriate index for glomerular filtration rate?" Basic Nephrol. Acute Kidney Inj., pp. 3-20 (2012).

27. G. C. Geddes, Y. M. Woo, and S. Brady, "Glomerular filtration rate: what is the rationale and justification of normalizing GFR for body surface area," Neprol. Dial. Transplant. 23, 4-6 (2007).

Martin P. Debreczeny is a consultant to MediBeacon Inc. and has led the device development efforts at MediBeacon since 2007. He has been researching new noninvasive optical medical devices for 20 years. He is also cofounder and managing member of BugLab LLC, which designs and manufacturers optical instruments for biofermentation monitoring. He is a coauthor on over 20 technical articles and coinventor on over 20 issued US patents.

Richard B. Dorshow is a cofounder, president, and chief scientific officer of MediBeacon Inc. and is a research physicist and technology manager with over 30 years in R\&D focusing on business oriented research programs. He is author on over 80 technical articles, and is coinventor on over 60 issued US patents. Currently, he is guiding MediBeacon through its clinical program to commercialize optical agents for human disease detection and monitoring. 